critical for a successful cure. Central to our strategy is to focus HIV-1-specific CTL on the most functionally conserved regions (not a string of epitopes and not full-length proteins) common to most HIV-1 variants, which HIV-1 typically cannot change without losing fitness. These conserved regions were defined by bioinformatics, and a bivalent mosaic was computationally designed to increase the vaccine perfect match of potential T-cell epitopes to $80 \%$ of global HIV-1 variants; if successful, the vaccine will be suitable for global deployment. Furthermore, we maximised the inclusion of protective epitopes associated with viral control in treatment-naive HIV1-positive individuals defined on 4 continents. These immunogens are delivered by the non-replicating simian adenovirus ChAdOx1 prime and non-replicating poxvirus MVA boost regimen, clinically proven safe and potent.

Methods The GREAT consortium has been established to build capacity for a future efficacy trial in Zambia, around Lake Victoria and in Kenya by engaging populations at documented high risk for HIV-1 infection, despite preventive interventions, by diverse clades. We will conduct a phase I/IIa clinical trial HIV-CORE 006 to assess the safety and immunogenicity of the conserved-region vaccines, in preparation for an efficacy trial in these at-risk populations.

Conclusion The aims of the GREAT consortium are to ensure that at the completion of the programme grant, the vaccine regimen will be proven safe and potent, and the sites will be prepared to launch an appropriately designed trial to prove vaccine efficacy.

\section{OC 8510 BIOTRANSFORMATION OF PRAZIQUANTEL FOR THE PHARMACOKINETIC OPTIMISATION OF PRAZIQUANTEL USE IN MASS DRUG ADMINISTRATION AND DEVELOPMENT OF NEW PAEDIATRIC FORMULATIONS}

${ }^{1}$ Roslyn S Thelingwani* ${ }^{1}$ Nyasha Kapungu, ${ }^{2}$ Xueging Li, ${ }^{1}$ Comfort Kanji, ${ }^{1}$ Chenai Mutiti ${ }^{3}$ Wilma J Reinhard-Rupp, ${ }^{1}$ Collen Masimirembwa. ${ }^{1}$ African Institute of Biomedical Science and Technology, Harare, Zimbabwe; ${ }^{2}$ AstraZeneca, Södertälje Sweden; ${ }^{3} E M D$ Serono, Darmstadt, Germany

\subsection{6/bmjgh-2019-EDC.26}

Background Praziquantel (PZQ) is the only drug available for the treatment of all forms of schistosomiasis. New paediatric formulations for the active enantiomer R-PZQ and the racemate PZQ are currently under development. There is however limited drug metabolism and pharmacokinetic data on PZQ available to support these initiatives. Detailed knowledge of PZQ metabolism will enable the use of PBPK modelling to determine appropriate doses for the new formulations in paediatric patients and to predict risks for drug-drug interactions in mass drug administration.

Methods Biotransformation studies on PZQ were conducted in human liver microsomes and recombinant Cytochrome P450s (CYPs). Structure elucidation was inferred from mass spectra. Enzyme kinetic studies to determine the Michaelis-Menten kinetics, $\mathrm{Km}$ and Vmax, of the formation of the main metabolites and analysis of clinical samples were determined by LCMS/MS.

Results CYP reaction phenotyping studies with HLM and $r$-CYPs indicate major involvement of CYP1A2, 2 C19, 2D6 and $3 \mathrm{~A} 4 / 5$ in the metabolism of $\mathrm{R}-$ and S-PZQ.
Biotransformation studies showed that PZQ is metabolised to cis-4-OH-PZQ mainly by CYP1A2 and CYP2C19. CYP3A4/5 metabolises PZQ to a mono-hydroxyl metabolite (X-OH-PZQ) whilst CYP2D6 metabolises PZQ to minor novel monohydroxyl metabolite (Y-OH-PZQ) both pending structural elucidation by nuclear magnetic resonance. R-PZQ was more rapidly cleared than S-PZQ with variable interindividual AUC and Cmax.

Discussion and conclusion The differential role of CYP1A2 and CYP2C19 and of CYP3A4 and CYP3A5 in the formation the 4-OH-PZQ and the novel $\mathrm{X}-\mathrm{OH}-\mathrm{PZQ}$ respectively are intriguing findings as this has not been reported before in humans. In vitro, cis and not trans 4-OH-PZQ formation has been observed contrary in vivo reports in humans which indicate trans 4-OH-PZQ as the main metabolite. The data will enable us to understand the rapid clearance of PZQ and predict potential drug-drug-gene interactions which may explain the inter-individual variability of PZQ pharmacokinetics.

\section{OC 8526 THE RWANDA CLINICAL RESEARCH NETWORK (RWANDA-CRN): A MODEL FOR MIXED SOUTH-SOUTH AND NORTH-SOUTH COLLABORATIONS FOR CLINICAL RESEARCH CAPACITY DEVELOPMENT}

Jean Pierre Musabyiman*, Clarisse Musanabaganwa, Valentine Dushimiyimana, Jean Pierre Namabajimana, Prosper Karame, Ladislas Nshimiyimana, Pacific Ndishimye. Rwanda Biomedical Center, Ministry of Health, Kigali, Rwanda

\subsection{6/bmjgh-2019-EDC.27}

Background Poor countries carry 90\% of the global burden of disease, with access to only $10 \%$ of globally available health research funding and technical capacity. Fragile southsouth collaborations hinder effective use of limited resources, career opportunities and funding to retain the insufficiently available quality scientists. The Rwanda Health System established a clinical research network involving academia, nongovernmental organisations and private sector to accelerate generation of talented scientists, create enabling environment and incentives to retain scientists by establishing a local funding model.

Methods Based on a baseline assessment, potential clinical trial units were mapped and developed through adoption of a Clinical trial management training model from European Universities. The Rwandan law on Public Private Partnership was leveraged to attract and engage local and international private players in a win-win approach. So far, countries such as Kenya and Sweden were engaged in the roadmap.

Results From 2014 to date, a total of 285 scientists are trained on various clinical research components: Good Clinical Practice (28\%), Research Grant writing (14\%), systematic review and meta-analysis (9\%) and scientific communication (8.7\%). Ten Clinical Research Units and one centre for evidence-based healthcare were established. So far 13 health investigator-initiated projects in malaria, metabolic disorders and maternal health were funded through the local funding model. A process to empower six malaria sentinel sites into fully functioning clinical research sites is underway.

Conclusion The creation of strong networks of excellence for clinical research among southern academic, research 